\title{
A Prospective, Population-Based Study of Trends in Operative Vaginal Delivery Compared to Cesarean Delivery Rates in Low- and Middle-Income Countries, 2010-2016
}

\author{
Margo S. Harrison, MD ${ }^{1}$, Sarah Saleem, MBBS$^{2}$, Sumera Ali, MBBS ${ }^{2}$, Omrana Pasha, MD², \\ Elwyn Chomba, MD ${ }^{3}$, Waldemar A. Carlo, MD ${ }^{4}$, Ana L. Garces, MD $^{5}$, Nancy F. Krebs, MD ${ }^{1}$, \\ K. Michael Hambidge, MD ${ }^{1}$, Shivaprasad S. Goudar, MD $^{6}$, S. M. Dhaded, MD ${ }^{6}$, Bhala \\ Kodkany, $\mathbf{M D}^{6}$, Richard J. Derman, MD $^{7}$, Archana Patel, MD, PhD $^{8}$, Patricia L. Hibberd, MD, \\ PhD $^{9}$, Fabian Esamai, MD ${ }^{10}$, Edward A. Liechty, MD $^{11}$, Antoinette Tshefu, MD ${ }^{12}$, Adrien \\ Lokangaka, MD ${ }^{12}$, Melissa Bauserman, MD $^{13}$, Carl L. Bose, MD $^{13}$, Fernando Althabe, MD ${ }^{14}$, \\ Jose M. Belizan, MD ${ }^{14}$, Pierre Buekens, MD ${ }^{15}$, Janet L. Moore, MS ${ }^{16}$, Dennis D. Wallace, \\ PhD $^{16}$, Menachem Miodovnik, MD ${ }^{17}$, Marion Koso-Thomas, MD ${ }^{17}$, Elizabeth M. McClure, \\ PhD $^{16}$, and Robert L. Goldenberg, MD ${ }^{18}$
}

${ }^{1}$ University of Colorado School of Medicine, Denver, Colorado ${ }^{2}$ Department of Community Health Sciences, Aga Khan University, Karachi, Pakistan ${ }^{3}$ University Teaching Hospital, University of Zambia, Lusaka, Zambia ${ }^{4}$ University of Alabama at Birmingham, Birmingham, Alabama ${ }^{5}$ Unidad de Planificación Instituto de Nutrición de Centroamérica y Panamá (INCAP), Guatemala City, Guatemala ${ }^{6}$ KLE University's Jawaharlal Nehru Medical College, Belgaum, India ${ }^{7}$ Thomas Jefferson University, Philadelphia, Pennsylvania ${ }^{8}$ Lata Medical Research Foundation, Nagpur, Maharashtra, India ${ }^{9}$ Boston University, Boston, Massachusetts ${ }^{10}$ Moi University School of Medicine, Eldoret, Kenya ${ }^{11}$ Indiana University School of Medicine, Indianapolis, Indiana ${ }^{12}$ Kinshasa School of Public Health, Kinshasa, Democratic Republic of the Congo ${ }^{13}$ Division of Neonatal-Perinatal Medicine, Department of Pediatrics, University of North Carolina School of Medicine, Chapel Hill, North Carolina ${ }^{14}$ Institute for Clinical Effectiveness and Health Policy, Buenos Aires, Argentina ${ }^{15}$ Tulane School of Public Health and Tropic Medicine, New Orleans, Louisiana ${ }^{16} \mathrm{RTI}$ International, Durham, North Carolina ${ }^{17}$ Eunice Kennedy Shriver, National Institute of Child Health and Human Development, Rockville, Maryland ${ }^{18}$ Department of Obstetrics/Gynecology, Columbia University, New York, New York

\author{
Abstract \\ Address for correspondence Margo S. Harrison, MD, MPH, Mail Stop B198-2, 12631 East 17th Avenue, Room 4211, Aurora, CO \\ 80045 (margo.harrison@ucdenver.edu). \\ Authors' Contributions \\ M.S.H. and R.L.G. conceived the analyses plan and drafted the first manuscript draft. W.A.C., C.L.B., E.M.M., D.D.W., N. F.K., \\ K.M.H., A.L.G., P.B., and F.E. edited the manuscript. S.S., O.P., E.C., W.A.C., A.L.G., N.F.K., K.M.H., S.S.G., B.K., R.J.D., A.P., \\ P.L.H., F.E., E.A.L., A.T., A.L., C.L.B., F.A., J.M.B., P.B., M.M., M.K.T., E.M.M., and R.L.G. developed the protocol. S.S., S.A., \\ E.C., A.L.G., S.S.G., S.M.D., A.P., F.E., A.L., A.T., and F.A. oversaw the study implementation. J.L.M., E.M.M., and D.D.W. \\ conducted the study analyses. All authors reviewed and approved the final manuscript. \\ Condensation \\ OVD (forceps- and vacuum-assisted vaginal delivery) is decreasing in the LMICs, while cesarean delivery rates are increasing. \\ Conflict of Interest \\ None.
}


Objective-Few data are available on cesarean delivery and operative vaginal delivery trends in low- and middle-income countries. Our objective was to analyze a prospective population-based registry including eight sites in seven low- and middle-income countries to observe trends in operative vaginal delivery versus cesarean delivery rates over time, across sites.

Study Design-A prospective population-based study, including home and facility births among women enrolled from 2010 to 2016, was performed in communities in Argentina, Democratic Republic of Congo, Guatemala, India, Kenya, Pakistan, and Zambia. Women were enrolled during pregnancy and delivery outcome data were collected.

Results-We analyzed 354,287 women; 4,119 (1.2\%) underwent an operative vaginal delivery and 45,032 (11.2\%) delivered by cesarean. Across all sites with data for 7 years, rates of operative vaginal delivery decreased from 1.6 to $0.3 \%$, while cesarean delivery increased from 6.4 to $14.4 \%$. Similar trends were seen when individual country data were analyzed. Operative vaginal delivery rates decreased in both hospitals and clinics, except in the hospital setting at one of the Indian sites.

Conclusion-In low- and middle-income countries, operative vaginal delivery is becoming less utilized while cesarean delivery is becoming an increasingly common mode of delivery.

\section{Keywords}

operative vaginal delivery; cesarean delivery; mode of delivery; low- and middle-income countries; facility-based delivery

In high-income countries (HIC), cesarean delivery (CD) rates are increasing with a concurrent decrease in operative vaginal delivery (OVD) rates. ${ }^{1-3}$ OVD includes both forceps and vacuum-assisted vaginal delivery. OVD is an essential component of emergency obstetrical care because it is used to expedite delivery in cases of fetal distress, to assist in situations of maternal exhaustion in labor, and to facilitate delivery when the patient has arrested in the second stage of labor, which is associated with adverse pregnancy outcomes when prolonged. ${ }^{4}$ Additionally, OVD avoids the alternative delivery option of $\mathrm{CD}$, which carries risks associated with major abdominal surgery, and may be available in settings, such as homes or clinics, where CD is not. ${ }^{4}$ Few data are available on these trends in low- and middle-income countries (LMIC), but it is known that availability of operative delivery supplies and health-care providers trained to perform OVD in these settings is limited. ${ }^{4}$

A cross-delivery health facility assessment of OVD in 40 LMIC in Latin America (LA), subSaharan Africa (SSA), and Asia reviewed the percentage of facilities where health workers performed OVD in the prior 3 months. ${ }^{4}$ Fewer than $20 \%$ of hospitals in LA and 53\% of hospitals in SSA (only $6 \%$ of health centers) had performed OVD during this time. ${ }^{4}$ In Asia, rates ranged from 5\% in Nepal to $89 \%$ in Afghanistan. ${ }^{4}$ The authors concluded that OVD is underused in countries where pregnant women continue to face hardships accessing $\mathrm{CD}$, and where $\mathrm{CD}$ can be relatively unsafe due to poor surgical technique and lack of resources. ${ }^{4}$ Unfortunately, no global consensus or recommendation has been established on the appropriate rate of OVD in a given population. In 2013, the American College of Obstetricians and Gynecologists reported a rate of 3\% in the United States, while the Royal 
College of Obstetricians and Gynaecologists reported a rate of 10 to $13 \%$ in the United Kingdom in 2011.5,6

Our objective was to analyze a prospective population-based pregnancy outcome registry from eight sites in seven LMICs to observe trends in OVD versus CD rates over time. We also evaluated the relationship between OVD and CD on a site-by-site basis. We hypothesized that CD rates would increase and OVD would decrease, suggesting that at least part of the rise in CD in LMIC might be explained by the decrease in forceps and vacuum, as has occurred in some HIC. We also wished to observe the trend by type of facility; we hypothesized that OVD would decrease overall, but particularly in clinic as compared with hospital settings.

\section{Materials and Methods}

These eight sites conducted the study as part of the Global Network for Women's and Children's Health Research (GN), a network of institutions that conducts research aimed at improving maternal and newborn outcomes. RTI International serves as the data coordinating center. The Eunice Kennedy Shriver National Institute of Child Health and Human Development (NICHD) in the United States funds the GN.

This analysis was conducted using data from a prospective study conducted in communities at eight sites in seven low-income countries on births from January 1, 2010 through December, 2016 (Corrientes, Argentina; Equateur, the Democratic Republic of the Congo [DRC]; Chimaltenango, Guatemala; Nagpur, and Belagavi, India; western Kenya; Thatta District, Pakistan; and Lusaka, Zambia). The DRC site initiated enrollment in 2014, while the Argentine site ceased enrollment mid-2012; because data for the full timeframe was not available, these two sites were excluded from the overall trend data.

The GN's prospective registry, the Maternal and Newborn Health Registry (MNHR), includes outcomes from rural or semiurban geographical areas. Each site includes between 6 and 24 distinct communities. ${ }^{7}$ Each community generally represents the catchment area of a primary health-care center, and $\sim 300$ to 500 births take place annually in each community. The objective of the MNHR is to enroll pregnant women as early as possible during the pregnancy and to obtain data on pregnancy outcomes for all deliveries of registered women, regardless of birth location (i.e., home, health clinic, or hospital). Each community employs a registry administrator who identifies, enrolls, and tracks pregnant women and their outcomes in coordination with community elders, birth attendants, and other health-care workers.

The MNHR can be used to quantify and analyze trends in pregnancy outcomes in defined geographic areas over time to provide population-based statistics on pregnancy outcomes, including stillbirths, and neonatal and maternal morbidity and mortality. The analyses presented here used the MNHR to determine trends in mode of delivery, comparing rates of $\mathrm{CD}$ to those of OVD across study sites over time. The MNHR question regarding delivery mode that was used in this study to define use of OVD changed slightly over the time period covered by this study. Prior to 2014, the question response of "Vaginal, assisted" was used to 
define OVD; subsequently, "Vaginal, with forceps/vacuum" was used. Because we were concerned that the registry administrators may have called some vaginal breech deliveries "assisted deliveries," we excluded breech and other malpresentations from the final analysis.

The population studied included women screened for the MNHR who were eligible, consented, and delivered between January 2010 and December 2016. Data were excluded from women who were enrolled but lost to follow-up prior to delivery, maternal deaths prior to labor and delivery, miscarriages, medically terminated pregnancies, pregnancies complicated by breech or other malpresentations, and those with missing data for delivery mode.

Data were collected and entered into research computers at each study site and transmitted through secure methods to a central data-coordinating center (RTI International). Counts and percentages of $\mathrm{CD}$ and OVD deliveries per GN site per year were obtained using standard contingency table techniques. Estimates of overall trends excluded data from Argentina (2010-2012) and the DRC (2014-2016) since these research sites did not have data collected for the full period of interest, 2010-2016. To generate point and interval estimates of the probability of a CD or OVD and to test for the presence of a trends over time, both by site and overall, we fit a binomial model with an identity link and used generalized estimated equations to control for the cluster-level effects. The model included terms for study site, year, and an interaction term for the site by year. A first-order orthogonal polynomial contrast was used to test for linear trend. Statistical analyses were done with SAS version 9.4 (SAS Institute, Cary, NC). Differences in hospital and clinic changes were examined descriptively with no formal statistical test conducted.

The appropriate institutional review boards/ethics research committees of the participating institutions approved the MNHR study. Individual informed consent for study participation is requested and obtained from each study participant. A Data Monitoring Committee, appointed by the NICHD, oversees and reviews the study semiannually. ${ }^{7}$

\section{Results}

Of 390,692 women screened from 2010 to $2016,384,887(99.7 \%)$ were consented and enrolled in the MNH registry study, and of those 354,287 (93.2\%) met inclusion criteria for this analysis; 3,797 (1.2\%) underwent an OVD and 36,782 (11.2\%) were delivered by CD (Fig. 1). From 2010 to 2016 across all sites excluding DRC and Argentina, rates of OVD decreased from 1.6 (95\% confidence interval [CI]: $1.2-2.0$ ) to $0.3 \%$ (95\% CI: 0.2-0.4), while overall CD rates increased from 6.4 (95\% CI: 5.6-7.3) to 14.4\% (95\% CI: 12.9-15.8) (Fig. 2 ). A test of trend for each method of delivery found a statistically significant change over time at $\boldsymbol{p}<0.0001$, when adjusted for site.

Because of the marked difference in rates of OVD and CD across regions, we also examined these by individual site. In Kenya, DRC, Pakistan, and Guatemala, the reduction in OVD over time was statistically significant $(p<0.05$ ), while trend test in other sites did not reach statistical significance. Regarding the increasing rates of CD over time, all sites except for 
the DRC and Zambia had statistically significant increases in CD rates over the period studied (Fig. 3).

We next evaluated the OVD use by type of health facility (excluding DRC and Argentina). Overall, facility births accounted for $72 \%$ of the births studied ( $N=234,855$ births), with $41 \%$ of facility births occurring in clinics and 59\% occurring in hospitals. Fig. 4 evaluates the trend in OVD rates by type of delivery institution. Rates of OVD in both hospital and clinic settings decreased over time in all sites except Belagavi.

\section{Discussion}

The principal finding of this analysis is that in LMIC, across regions including LA, SSA, and Southeast Asia, OVD rates decreased while CD rates increased, except in Belagavi where OVD rates increased from $0.5 \%$ in 2010 to $1.2 \%$ in 2016 in the hospital setting (but not the clinic setting). The decrease in OVD utilization was seen in both hospital and clinic settings in all other sites, even with facility birth becoming more common within the GN over time. Rates of both OVD and CD would be expected to rise with more births taking place in a setting with a skilled birth attendant.

Our analysis highlights the dwindling role of OVD across LMIC, echoing findings previously published from HIC. ${ }^{1-3}$ OVD is a safe and viable alternative to CD in the second stage of labor when it is used under appropriate circumstances by appropriately trained providers. ${ }^{5}$ This is particularly true in LMIC settings where CD may not be readily available but delivery needs to be expedited due to maternal and/or fetal risks. Avoiding CD can help prevent complications of the surgery such as hemorrhage and infection as well as longer term issues associated with future fertility such as adhesive disease, repeat CD, trial of labor after $\mathrm{CD}$, and abnormal placentation. ${ }^{5}$ The absolute risk of OVD to mothers and babies is quite low; OVD has been associated with perineal tearing and maternal urinary and fecal incontinence, but the data are inconsistent and long-term outcomes appear to be similar to spontaneous vaginal delivery. ${ }^{5}$ In terms of neonatal outcomes, vacuum and forceps have been associated with intracranial hemorrhage and neurologic complications, but not neonatal mortality. ${ }^{5}$ For these reasons, it is crucial that OVD be performed by well-trained providers. The fact the OVD is generally a safe and effective method to facilitate vaginal delivery, but rates are decreasing in LMIC, raises the question as to the reasons for decreased OVD utilization.

One explanation might be that patient and provider preference may have a role in the decision-making process regarding method of delivery. For example, many audits of institutions in LMIC have been published that review indications for CD as an attempt to better understand the rising $\mathrm{CD}$ rates. A Tanzanian audit found that unnecessary and nonevidence-based $\mathrm{CD}$ were performed in most cases, highlighting that OVD, and unassisted vaginal delivery, may not have been appropriately considered as alternative delivery options.

${ }^{8}$ A study from India suggested that nonindicated CD are performed for financial reasons, as the provider receives higher reimbursement from $\mathrm{CD}$ than vaginal delivery. ${ }^{9} \mathrm{In}$ a program where two Indian states paid providers directly to deliver poor women, the first by paying a certain amount per delivery by method (paying more for $\mathrm{CD}$ than vaginal delivery) and the 
second paying just per delivery ( $\mathrm{CD}$ and vaginal delivery were reimbursed the same), a very interesting result occurred. ${ }^{9}$ Where providers were reimbursed more for the $\mathrm{CD}$, the $\mathrm{CD}$ rate increased to $40.7 \%$ from a background rate of $4.9 \%$, whereas in the other state where providers were paid by delivery and not by delivery method, the CD rate decreased from a background rate of 8.1 to $4.3 \% .^{9}$ This example suggests that financial incentives and disincentives can significantly impact delivery method. Regarding patient preference, the apparent rise in cesarean deliveries could also be accounted for in part by CD performed by maternal request. A study from Nigeria found that $6.4 \%$ of patients reported willingness to request $\mathrm{CD}$; reasons women cited were fear of poor neonatal outcomes from vaginal birth and pain during the process. ${ }^{10}$ Conversely, a recently published paper from Argentina found that high $\mathrm{CD}$ rates are unlikely related to patient preferences for mode of delivery. ${ }^{11}$

There are also economic implications to our findings; it is far more resource intensive to perform a CD than an OVD. CD can lead to a catastrophic expenditure for women and their families, and can affect food purchasing and contribute to familial debt for years following the intervention. ${ }^{12}$ Another possible explanation that may account for lack of forceps and vacuum utilization in low-resource settings is lack of equipment used in OVD and the availability of trained staff. ${ }^{13}$

We suspect that rising CD rates may account, at least in part, for the decrease seen in OVD utilization, as has been stated by studies in HIC. ${ }^{5}$ For example, the American College of Obstetricians and Gynecologists in the United States states that the drop in OVD rates from 9 to 3\% from 1992 to 2013 accounts for part of the increase in CD rates in the United States. ${ }^{5}$ While this inverse relationship, seen in many settings, is not as stark in the SSA sites, we are concerned that there may be a focus on training providers, or at least a cultural shift in provider preferences, to perform $\mathrm{CD}$ to the exclusion of forceps and vacuum.

We believe that in regions such as SSA where overall rates of CD are often less than 3\%, maintaining OVD as a viable skill should be a priority. We suggest that national and global health organizations place an emphasis on the importance of continuing training in and encouraging the availability of forceps and vacuum delivery across facility settings, to ensure that both patients and providers have a range of emergency obstetrical care options available to them. ${ }^{14}$ Of note, the WHO is currently testing a new device called the Odon device as an alternative method to vacuum and forceps for assisted vaginal delivery, suggesting that the organization does value OVD as an alternative to $\mathrm{CD}$ and is researching and promoting its use. ${ }^{15}$

The strength of this study is that data are derived from a large, prospective population-based cohort that includes women from three continents with relatively consistent findings across regions. Limitations include the slight change in definition of OVD during the study, that we are not able to provide separate rates for forceps-assisted and vacuum-assisted vaginal deliveries, that some of the sites did not include data for the full time of interest, and that overall trends may be biased by sites with higher versus lower rates of OVD and CD, or an increasing number of deliveries in the facility setting. 
This analysis should bring attention to OVD as a dwindling, but still essential, component of emergency obstetrical care in LMIC. ${ }^{14}$ OVD offers some of the benefits of a vaginal delivery while avoiding some of the risks associated with $\mathrm{CD}^{5,14}$ We believe more resources should be invested in maintaining OVD as a safe option for labor and delivery care around the globe, and in LMIC specifically.

In LMICs, OVD is becoming a less utilized mode of delivery in both hospital and clinic settings, while $\mathrm{CD}$ is concurrently becoming an increasingly common mode of delivery.

\section{Acknowledgments}

Funding

This study was funded by grants from the Eunice Kennedy Shriver National Institute of Child Health and Human Development: U01 HD040477, U01 HD043475, U01 HD043464, U01 HD040657, U01 HD042372, U01 HD040607, U01HD040636, U01 HD040574, U01 HD40636.

\section{References}

1. Muraca GM, Sabr Y, Brant R, Cundiff GW, Joseph KS. Temporal and regional variations in operative vaginal delivery in Canada by pelvic station, 2004-2013. J Obstet Gynaecol Can 2016;38(07): 627-635 [PubMed: 27591346]

2. McQuivey RW. Vacuum-assisted delivery: a review. J Matern Fetal Neonatal Med 2004;16(03):171180 [PubMed: 15590444]

3. Notzon FC. International differences in the use of obstetric interventions. JAMA 1990;263(24): 3286-3291 [PubMed: 2348539]

4. Bailey PE, van Roosmalen J, Mola G, Evans C, de Bernis L, Dao B. Assisted vaginal delivery in low and middle income countries: an overview. BJOG 2017;124(09):1335-1344 [PubMed: 28139878]

5. Operative Vaginal Delivery. AGOG Practice Bulletin No 154, 112015

6. Operative Vaginal Delivery. RCOG Green-top Guideline No 26, 12011 Available at: https:// www.rcog.org.uk/globalas-sets/documents/guidelines/gtg_26.pdf. Accessed March 30, 2017

7. Goudar SS, Carlo WA, McClure EM, et al. The Maternal and Newborn Health Registry Study of the Global Network for Women's and Children's Health Research. Int J Gynaecol Obstet 2012;118(03): 190-193 [PubMed: 22738806]

8. Maaløe N, Bygbjerg IC, Onesmo R, Secher NJ, Sorensen BL. Disclosing doubtful indications for emergency cesarean sections in rural hospitals in Tanzania: a retrospective criterion-based audit. Acta Obstet Gynecol Scand 2012;91(09):1069-1076 [PubMed: 22642620]

9. Bogg L, Diwan V, Vora KS, DeCosta A. Impact of alternative maternal demand side financial support programs in India on cesarean sectiondelivery rates: indications of supplier-induced demand. Matern Child Health J 2016;20(01):11-15 [PubMed: 26259956]

10. Akintayo AA, Ade-Ojo IP, Olagbuji BN, Akin-Akintayo OO, Ogundare OR, Olofinbiyi BA. Cesarean section on maternal request: the viewpoint of expectant women. Arch Gynecol Obstet 2014;289(04):781-785 [PubMed: 24173172]

11. Mazzoni A, Althabe F, Gutierrez L, et al. Women's preferences and mode of delivery in public and private hospitals: a prospective cohort study. BMC Pregnancy Childbirth 2016;16:34 [PubMed: 26857448]

12. Arsenault C, Fournier P, Philibert A, et al. Emergency obstetric care in Mali: catastrophic spending and its impoverishing effects on households. Bull World Health Organ 2013;91(03):207-216 [PubMed: 23476093]

13. Manasyan A, Saleem S, Koso-Thomas M, et al.; EmONC Trial Group. Assessment of obstetric and neonatal health services in developing country health facilities. Am J Perinatol 2013;30(09):787794 [PubMed: 23329566] 
14. Gei AF. Prevention of the first cesarean delivery: the role of operative vaginal delivery. Semin Perinatol 2012;36(05):365-373 [PubMed: 23009970]

15. Requejo JH, Belizán JM. Odon device: a promising tool to facilitate vaginal delivery and increase access to emergency care. Reprod Health 2013;10:42 [PubMed: 23962177] 


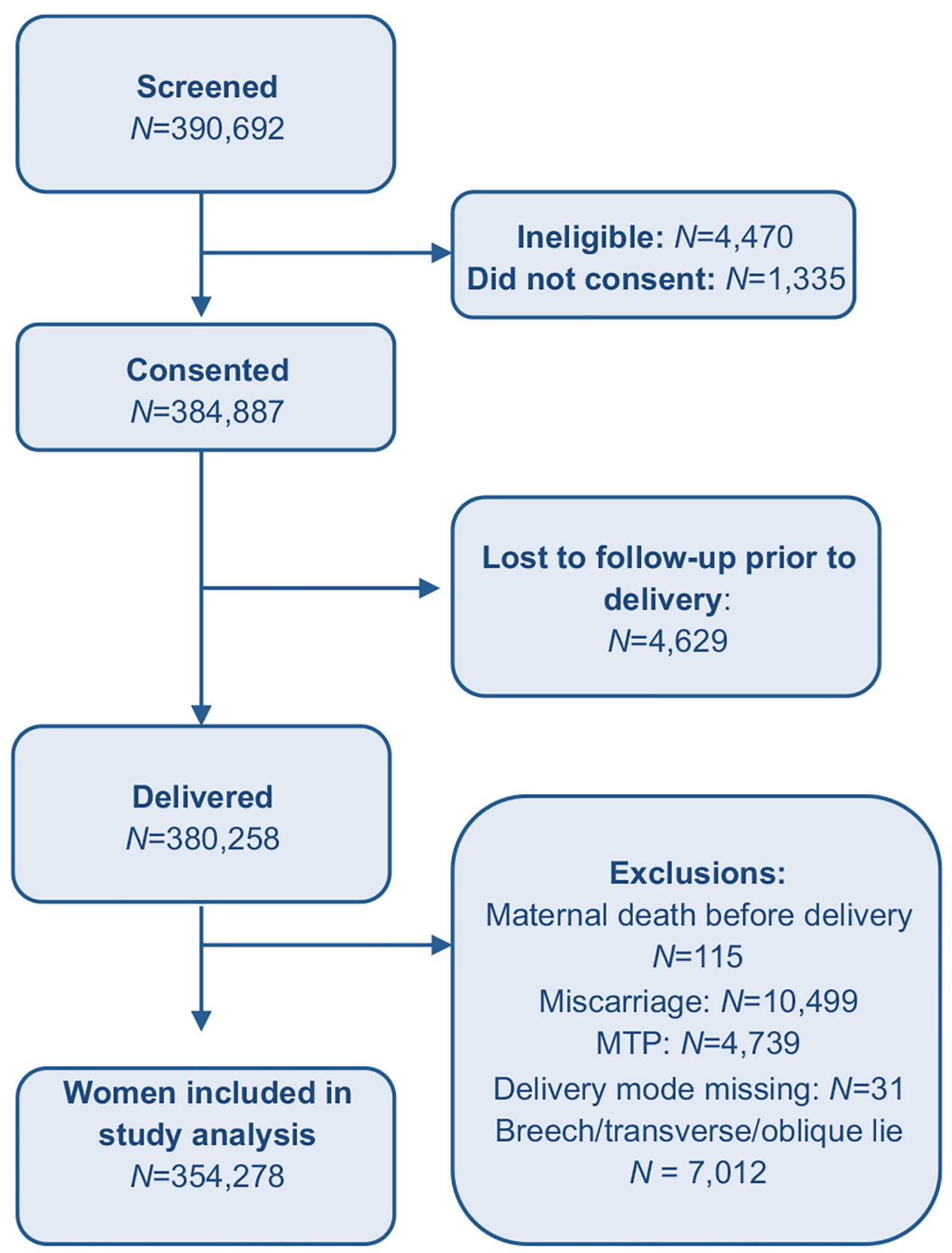

Fig. 1.

Enrollment diagram for Global Network Study on Delivery Mode, 2010 to 2016. 


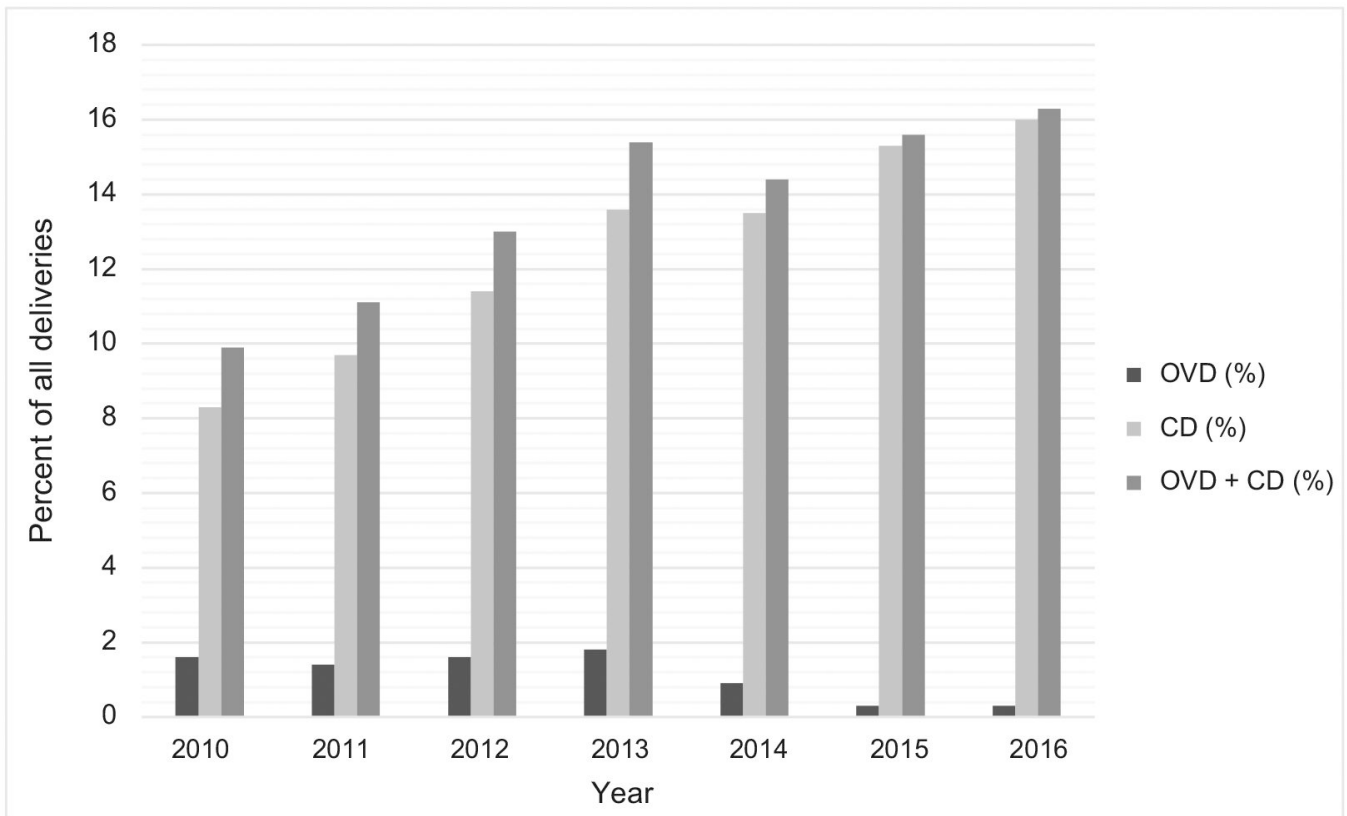

Fig. 2.

Rates (\%) of operative vaginal deliveries (OVD), cesarean deliveries (CD), and overall delivery rates $(O V D+C D)$ over time for all Global Network sites, 2010 to 2016. Trend test (2010-2016) for both CD and OVD. $p<0.0001$. 

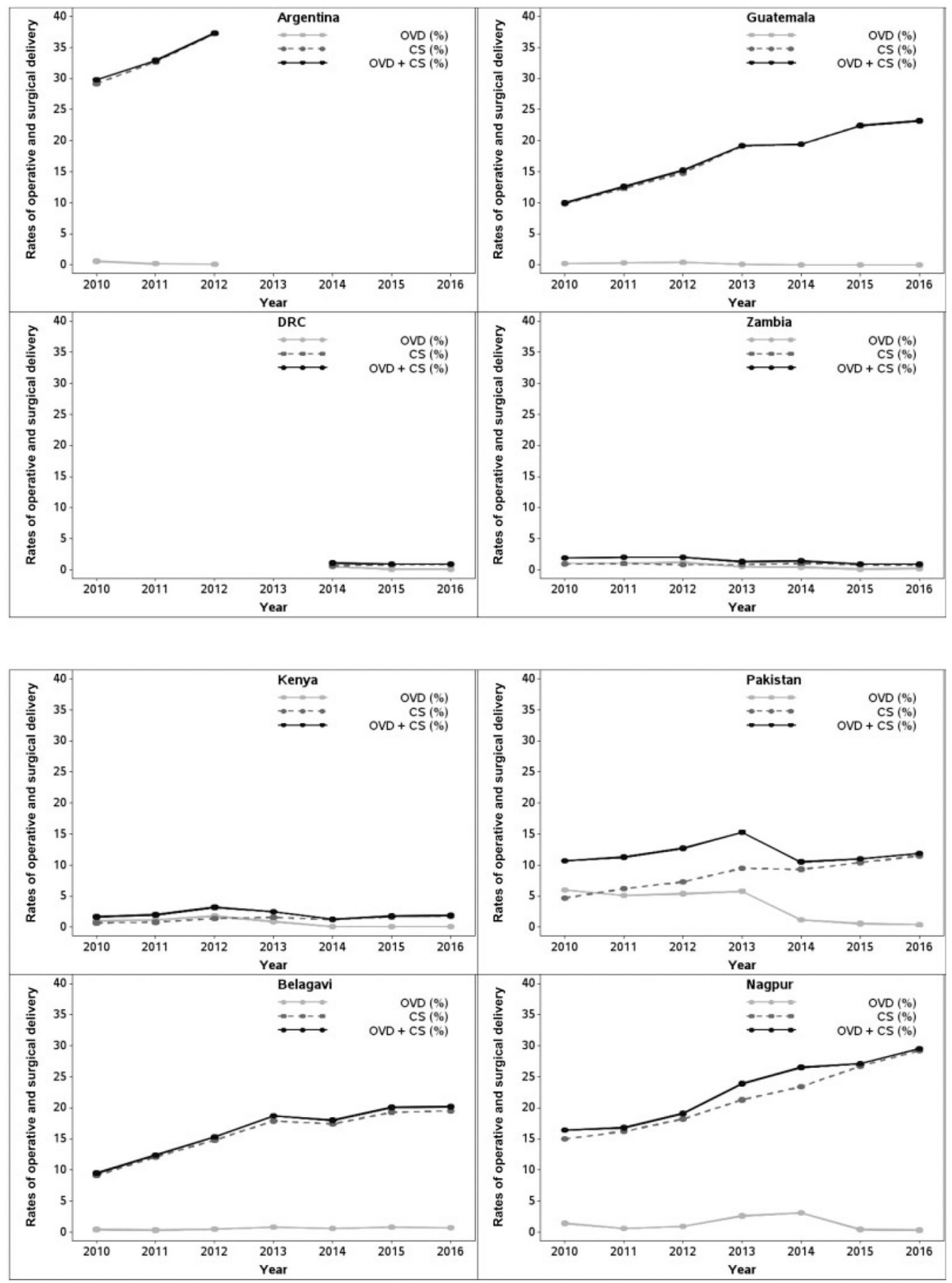

Fig. 3.

Rates (\%) of operative vaginal deliveries (OVD), cesarean deliveries (CD), and overall delivery rates (OVD + CD) over time by Global Network site, 2010 to 2016. 

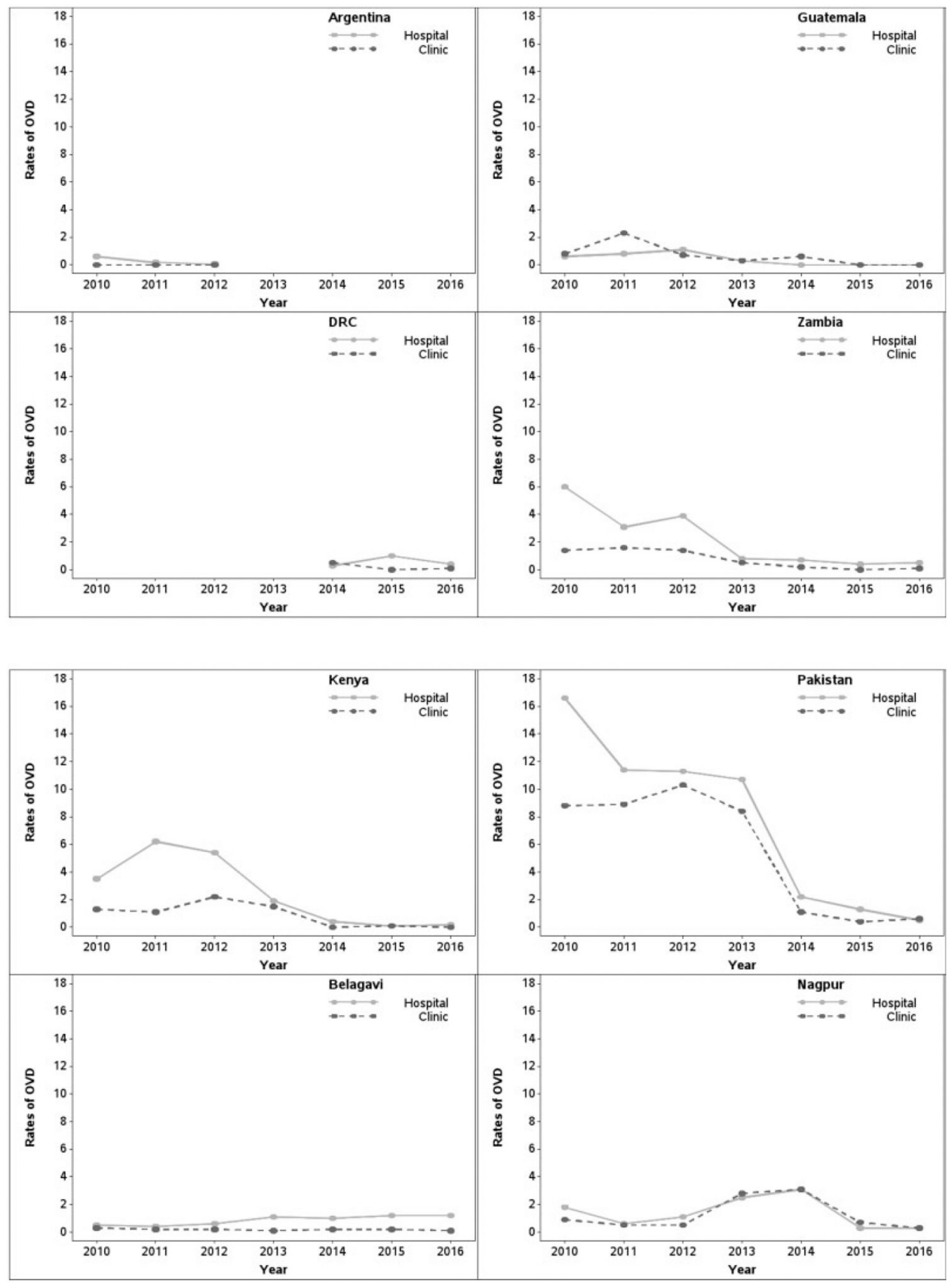

Fig. 4.

Rates (\%) of operative vaginal deliveries (OVD) by delivery location, Global Network sites 2010 to 2016. 